\title{
TETRALOGY OF FALLOT WITH ABSENT PULMONARY VALVE: SIMPLIFIED TECHNIQUE FOR HOMOGRAFT REPAIR
}

Paul M. Kirshbom, MD, James J. Jaggers, MD, and Ross M. Ungerleider, MD, Durham, NC

Tetralogy of Fallot (TOF) with absent pulmonary valve syndrome (APVS) occurs in $3 \%$ to $5 \%$ of cases of TOF. In addition to the ventricular septal defect, displaced infundibular septum, and the variable degree of pulmonary outflow tract obstruction that define TOF, patients with APVS also have dysplastic or entirely absent pulmonary valve leaflets. The main and branch pulmonary arteries of these patients are dilated or, frankly, aneurysmal. This aneurysmal dilatation can lead to airway compression and respiratory distress.

Patients with APVS can be divided into 2 groups: children with respiratory difficulties during the neonatal period and children with less airway compromise who tend to be identified later in life. Those patients who are identified later have a variable course with either congestive heart failure or the classic cyanotic spells seen in patients with TOF, depending on the degree of right ventricular outflow obstruction. This older group can undergo repair on an elective basis with good results. ${ }^{1-3}$

Patients with respiratory difficulties as neonates typically have a complicated course with recurrent airway problems

From the Department of Surgery, Duke University Medical Center, Durham, NC.

Received for publication July 30, 1999; accepted for publication Aug 11, 1999.

Address for reprints: Ross M. Ungerleider, MD, Box 3178, Duke University Medical Center, Durham, NC 27710 (E-mail: unger002@mc.duke.edu).

J Thorac Cardiovasc Surg 1999;118:1125-7

Copyright (C) 1999 by Mosby, Inc.

$0022-5223 / 99 \$ 8.00+0 \quad \mathbf{1 2 / 5 4 / 1 0 2 0 8 7}$ and pneumonias. Numerous methods of repair have been reported in the literature, most of which have included variations on pulmonary artery plication with or without inclusion of a neopulmonary valve. ${ }^{1-5}$ This report discusses a simplified technique for repair of APVS that we believe will yield more consistent results and better outcomes for this difficult patient population.

Patients and methods. Two neonates with APVS underwent surgical repair at Duke University Medical Center between November 1998 and April 1999.

PATIENT 1. A baby girl was brought to a regional medical center with respiratory distress on November 25,1998 , at $2 \frac{1}{2}$ weeks of age. The patient's distress and arterial oxygen desaturations progressed, necessitating intubation and transfer to Duke University Medical Center on the following day. The baby underwent diuresis, and an echocardiogram was obtained. This study revealed the classic findings of TOF with APVS. The pulmonary anulus was $5.5 \mathrm{~mm}$ in diameter with mild stenosis and severe insufficiency. The main pulmonary artery was 1.3 to $1.5 \mathrm{~cm}$ in diameter with markedly dilated branch pulmonary arteries. Cardiac catheterization confirmed these findings and demonstrated the typical small, spindly branch pulmonary arteries.

The baby underwent surgical repair on December 1, 1998. She underwent extubation on postoperative day 3 and was discharged in good condition on day 11. Follow-up echocardiography confirmed a widely patent right ventricular outflow tract and only trace pulmonary insufficiency.

PATIENT 2. A baby girl was a 3424-g product of an uncomplicated term pregnancy who was noted to be cyanotic soon after birth. Arterial $\mathrm{Po}_{2}$ was $34 \mathrm{~mm} \mathrm{Hg}$ on $100 \%$ oxygen. 


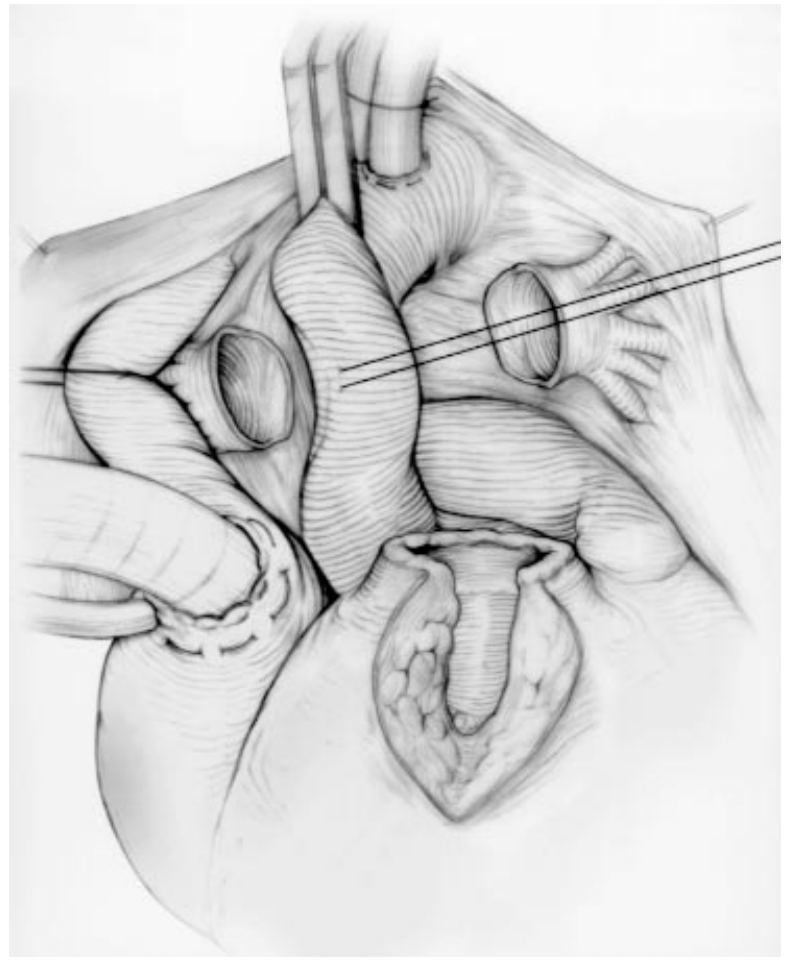

Fig 1. The main and branch pulmonary artery tissue is removed entirely, leaving "cuffs" of pulmonary artery from which the lobar/segmental branches arise.

Echocardiography revealed TOF with APVS and markedly dilated main and proximal branch pulmonary arteries. There was a 43-mm $\mathrm{Hg}$ gradient across the pulmonary anulus.

The patient's respiratory status improved with diuresis, although she continued to experience intermittent cyanotic episodes. The patient underwent surgical repair at 9 days of age. Postoperative inotropic support with dopamine and epinephrine was weaned by day 3 , and she underwent extubation on postoperative day 5 . The remainder of the postoperative course was unremarkable, with discharge on day 10.

Technique. After routine median sternotomy and placement on cardiopulmonary bypass, the left and right pulmonary arteries are dissected out to the lobar branches. The patient's body is cooled to $25^{\circ} \mathrm{C}$, and the ventricular septal defect is closed with a polytetrafluoroethylene patch through a right ventriculotomy. The main and branch pulmonary arteries are then resected, leaving only a cuff of pulmonary artery tissue around the takeoff of the lobar branches (Fig 1). The back walls of the left and right pulmonary artery cuffs are then approximated in the midline with a continuous suture (Fig 2). This is fairly easy to accomplish in a neonate because the distance that the pulmonary arteries need to be mobilized is fairly short. This technique effectively removes all abnormal main and branch pulmonary artery tissue and leaves only the pulmonary artery cuffs from which the lobar/segmental

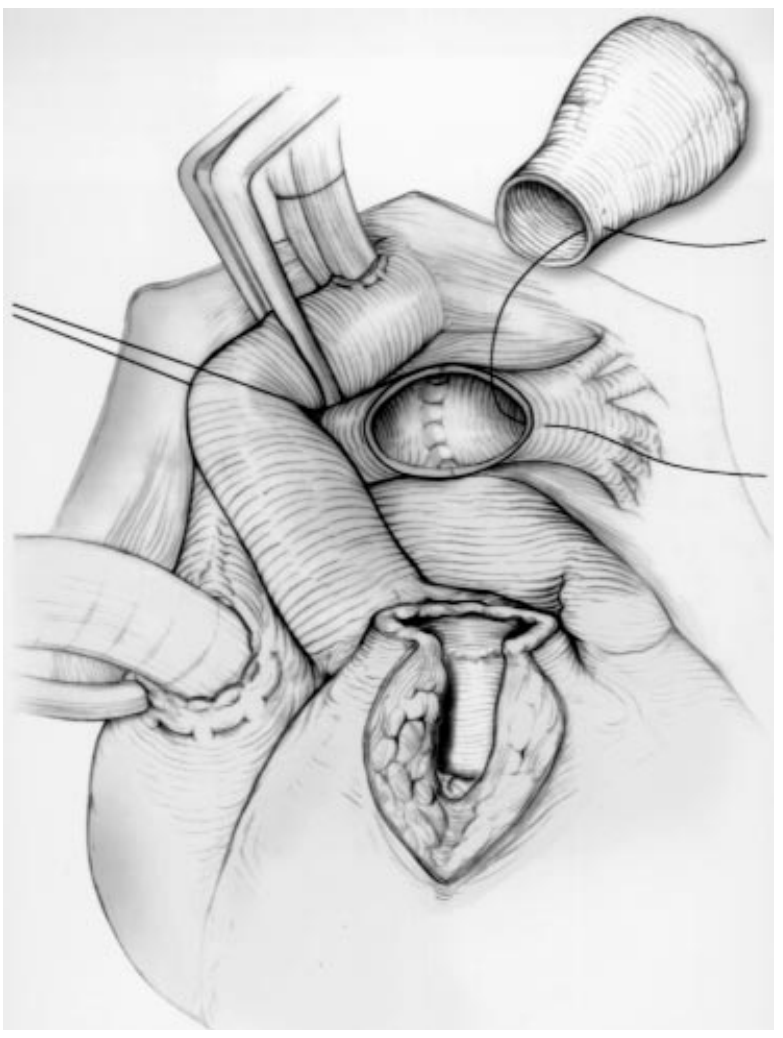

Fig 2. With the aorta retracted laterally, the posterior aspects of the pulmonary artery "cuffs" are anastomosed to create a new pulmonary artery bifurcation. A pulmonary homograft is then anastomosed distally.

pulmonary arteries arise. An appropriate-sized homograft is anastomosed to the neopulmonary artery distally (Fig 3) and the right ventricle proximally in the standard fashion. Removal of the crossclamp, rewarming, and decannulation proceed in the usual manner.

Comment. TOF with APVS can present a difficult and frustrating clinical problem for surgeons, cardiologists, and pediatricians. The patients with this lesion who have respiratory compromise early in life typically have a complicated course with recurrent respiratory difficulties despite surgical repair of the primary lesion. These recurrent problems may be due to irreversible changes in the airways (bronchomalacia or tracheomalacia) believed to be the result of compression during prenatal and neonatal development. It is unclear whether current surgical techniques are entirely successful in relieving the primary disease process. There are 2 main points that differentiate the many techniques proposed for repair of this lesion. First is the method of pulmonary artery resection or plication, and second is whether a pulmonary valve is inserted or created.

The method presented here is a modification of several existing techniques including the pulmonary resection/arterioplasty with monocusp insertion proposed by Kreutzer and 


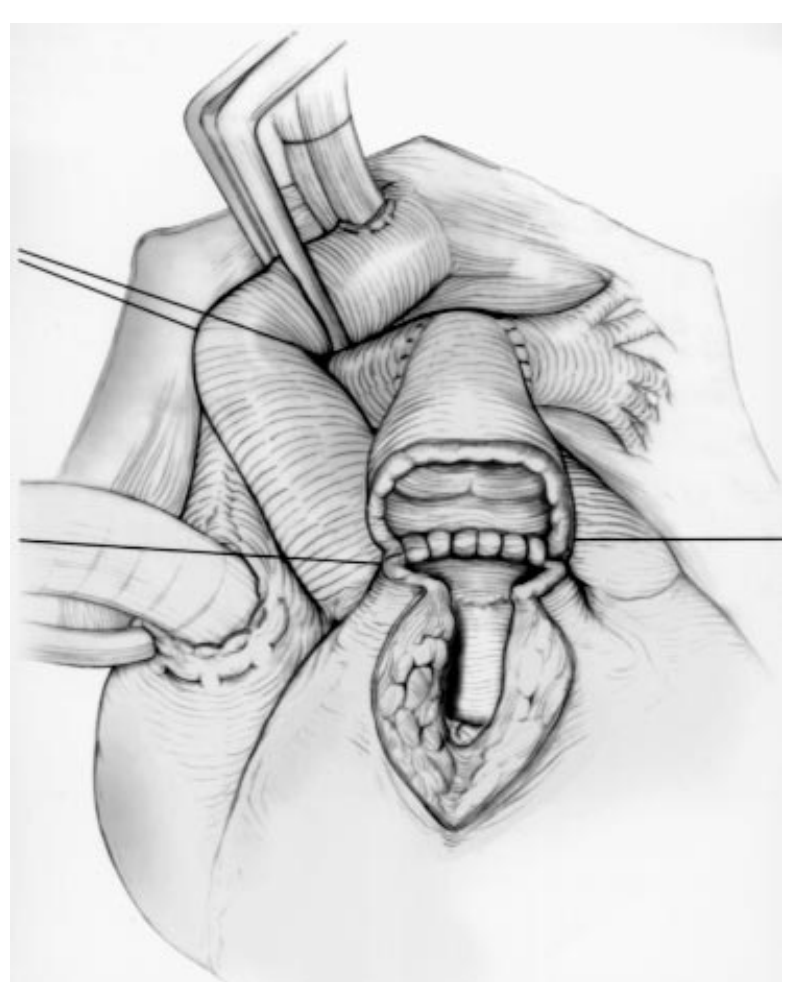

Fig 3. The proximal pulmonary homograft anastomosis is performed to the distal aspect of the right ventricular outflow tract.

colleagues $^{5}$ and the homograft repair reported by Snir and colleagues. ${ }^{1}$ The main advantage of this technique is the elimination of essentially all of the abnormal pulmonary arte- rial tissue with insertion of a valved conduit. Although many groups $^{2}$ continue to recommend plication, these techniques can be complicated, variable, and often less than satisfactory because they can produce recurrent dilatation or kinking in the postoperative period.

Although some suggest that good results can be achieved without the need for a homograft, ${ }^{1,4}$ we believe that at the current time a pulmonary homograft offers the most reliable prospect for creating a near-normal pulmonary outflow tract in this group of patients who will likely have numerous pulmonary problems during the first several years of life. Medium- and long-term follow-up certainly will be necessary before this technique can be considered superior to other options. However, this is a straightforward, reproducible technique that should be considered when one is faced with one of these difficult scenarios.

\section{REFERENCES}

1. Snir E, de Leval M, Stark J. Current surgical technique to repair Fallot's tetralogy with absent pulmonary valve syndrome. Ann Thorac Surg 1991;51:979-82.

2. McDonnell BE, Raft GW, Gaynor JW, et al. Outcome after repair of tetralogy of Fallot with absent pulmonary valve. Ann Thorac Surg 1999;67:1391-6.

3. Jekel L, Benatar A, Bennink GBWE, Woolley SR, Wal HJ. Tetralogy of Fallot with absent pulmonary valve: a continuing challenge. Scand Cardiovasc 1998;32:213-8.

4. Godart F, Houyel L, Lacour-Gayet F, et al. Absent pulmonary valve syndrome: surgical treatment and considerations. Ann Thorac Surg 1996;62:136-42.

5. Kreutzer C, Schlicter A, Kreutzer G. Tetralogy of Fallot with absent pulmonary valve: a surgical technique for complete repair. J Thorac Cardiovasc Surg 1999;117:192-4. 